\title{
The Evaluation of the Effectiveness of a Structured Educational Program on Nurses' Assessment Ratings in an Epilepsy Monitoring Unit
}

\author{
Mona Baran Stecker \\ West Virginia University
}

Follow this and additional works at: https://researchrepository.wvu.edu/etd

\section{Recommended Citation}

Baran Stecker, Mona, "The Evaluation of the Effectiveness of a Structured Educational Program on Nurses' Assessment Ratings in an Epilepsy Monitoring Unit" (2011). Graduate Theses, Dissertations, and Problem Reports. 3057.

https://researchrepository.wvu.edu/etd/3057

This Dissertation is protected by copyright and/or related rights. It has been brought to you by the The Research Repository @ WVU with permission from the rights-holder(s). You are free to use this Dissertation in any way that is permitted by the copyright and related rights legislation that applies to your use. For other uses you must obtain permission from the rights-holder(s) directly, unless additional rights are indicated by a Creative Commons license in the record and/ or on the work itself. This Dissertation has been accepted for inclusion in WVU Graduate Theses, Dissertations, and Problem Reports collection by an authorized administrator of The Research Repository @ WVU.

For more information, please contact researchrepository@mail.wvu.edu. 
The Evaluation of the Effectiveness of a Structured Educational Program on Nurses' Assessment Ratings in an Epilepsy Monitoring Unit

\author{
Mona Baran Stecker
}
Doctoral Research Project submitted to the School of Nursing at West Virginia University in partial fulfillment of the requirements for the degree of Doctor of Nursing Practice

\author{
Heidi Putman-Casdorph, PhD, RN, Chair \\ Susan McCrone, PhD, RN \\ Terrence Edward Patterson, PhD
}

Morgantown, West Virginia

2011

Keywords: education, nurse, epilepsy, monitoring, assessment 


\begin{abstract}
The Evaluation of the Effectiveness of a Structured Educational Program on Nurses' Assessment Ratings in an Epilepsy Monitoring Unit

Mona Baran Stecker
\end{abstract}

Background: There has been little published about nursing care on an epilepsy monitoring unit (EMU). Patients with epileptic and non-epileptic events require close monitoring and thorough assessments. In addition, it is especially important that these patients are provided a safe environment.

Purpose: The purpose of this project was to evaluate the impact of a structured educational program for nurses on an EMU in the assessment of patients with clinical events by quantitatively measuring nursing assessments before and after the program.

Objectives: There were two objectives for this project. The first objective was to measure nurses' assessments of patients on the EMU in a quantifiable form. The second objective was to evaluate the effectiveness of an educational program on the scores on the Nursing Assessment Rating Scale.

Design: The study used a before and after design to evaluate the effectiveness of an educational program on nurse assessment ratings. Nurses on the EMU attended one multi-modal educational session consisting of reinforcement of existing protocols and didactic lectures one of which used case-based scenarios with interactive participation.

Subjects: Twenty-Five nurses working on the EMU participated in the study.

Results: Nurses' assessments were quantified using a criterion based rating scale with seven primary quality indicators. The indicators were characterized as Superior (3 points), Good ( 2 points), Satisfactory (1 point) and Unsatisfactory( 0 points). Prior to the education, the mean scores on the rating scale were 14.5 points (SD 2.1). Post education, there was a statistical difference in scores 16. (SD1.6) $(\mathrm{p}<.01)$.

Conclusions: Three primary conclusions were reache. First, the Nurse Assessment Rating Scale was able to transform a qualitative measure of nursing assessment into a quantitative indicator that could be compared pre-and post-education. By assigning a numeric value to the rating of superior, good, satisfactory, or unsatisfactory, a quantitative score was compared to ascertain whether the education did improve scores. Second, the data indicated that the education significantly improved the assessment scores. With education, the original protocols were reinforced and assessments became more consistent resulting in improved scores. Third using a multimodal education format targeted aspects of performance such as respect and compassion. 


\section{Acknowledgements}

I owe a debt of gratitude to many people (some of who are deceased) who have, in various ways, helped me with this project. Words alone cannot express how grateful I am to those who showed patience in constructing this worthwhile endeavor.

*Many thanks to my committee members Dr. Heidi Putman-Casdorph, Dr. Susan McCrone, and Dr. Terry Patterson, who endlessly encouraged and guided me through this process. A special thanks to Dr. Putman-Casdorph who selflessly worked with me to bring this project to fruition.

*Thank you to my mother, Eileen Krubitzer (deceased) who was a wonderful role model and the most caring nurse I have ever known. While I can never hope fill her shoes, I hope that she is proud of this achievement.

*Thanks to my father William Krubitzer (deceased) who always thought I was special and gave me his support me through the years.

*A very special thank-you to my husband, Mark, without whom I could not have completed this project. His expertise in the field of epilepsy is second to none. He also believed in me when I did not believe in myself.

*Thank you to Carrie Cox, Marshall graduate student, who helped me with data collection and entry. 


\section{Table of Contents}

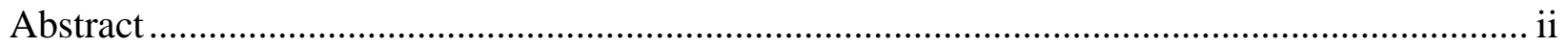

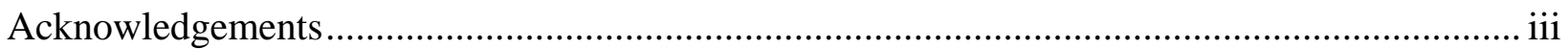

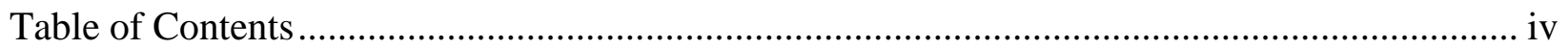

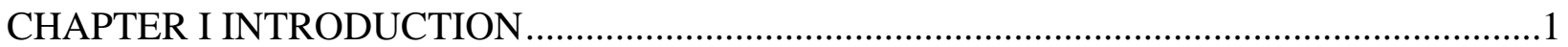

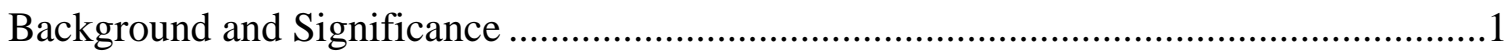

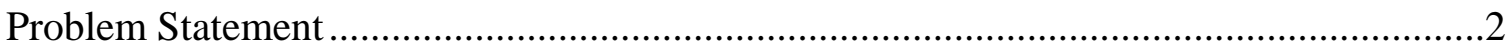

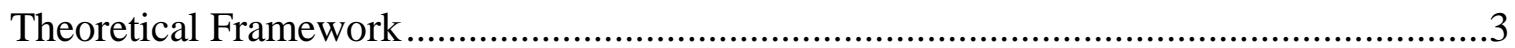

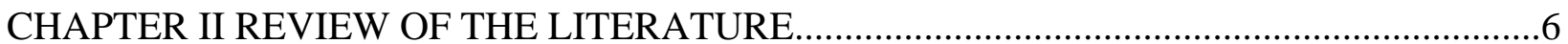

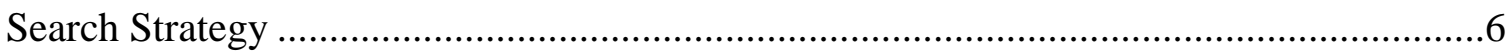

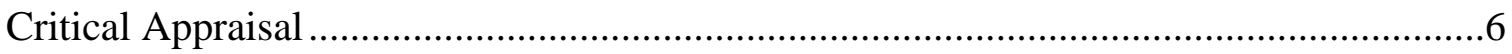

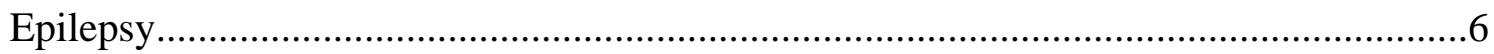

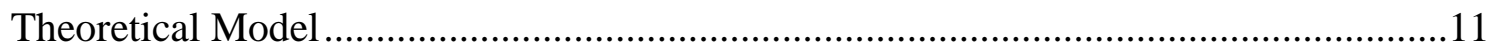

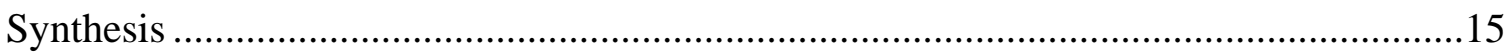

Congruence with Organizational Strategic Plan .......................................................16

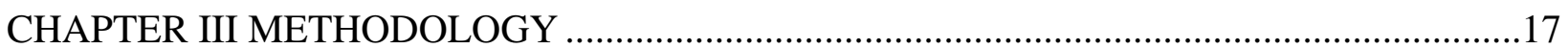

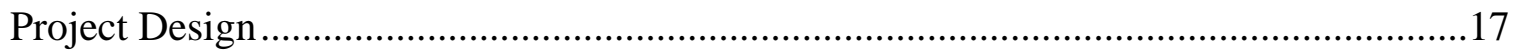


Educational Program.

Project Timeline.

Resources/Budget

Planned Evaluation

CHAPTER IV RESULTS

EMU Patient Demographics

EMU Nursing Demographics

Pre/Post Education Assessment Scores

Discussion

Conclusions

Limitations

References

Appendix A

Appendix B

Appendix C 


\section{Chapter I Introduction}

\section{Background and Significance}

Little has been published about nursing care on epilepsy monitoring units (EMUs). Patients with epileptic and non-epileptic events require close monitoring and thorough assessments. In addition, it is especially important that these patients are provided a safe environment.

Information from the World Health Organization (WHO, 2009) indicates that globally, 50 million people have epilepsy (WHO, 2009) and among those affected, $90 \%$ are from developing countries. The WHO also estimates that three fourths of people with epilepsy in developing countries are not treated for this disorder.

Epilepsy and seizures affect almost 3 million Americans of all ages, with an estimated annual cost of approximately 15.5 billion dollars (Epilepsy Foundation, 2010). Approximately 200,000 new cases of seizures and epilepsy occur each year (Epilepsy Foundation, 2010) and ten percent of the American population will experience a seizure in their lifetime (Pallin et al., 2008). Current estimates indicate that up to three percent of the adult population will develop epilepsy by the age of 75 (Epilepsy Foundation, 2010).

Of the three million people with epilepsy, 25-30\% do not attain adequate seizure control (National Association of Epilepsy Centers, 2010). It is estimated that seizures constitute one million visits to Emergency Departments annually, accounting for one percent of all ED visits in the U.S. (Pallin et al., 2008). 
As there is no standardized mechanism for tracking epilepsy in the state of West Virginia, data for incidence and prevalence are not available. In 2001, however, there were 101 deaths in West Virginia attributed to epilepsy which equaled $0.65 \%$ of the population (West Virginia Department of Health and Human Resources, 2002). Currently, there are only two epilepsy monitoring units in the state of West Virginia.

The literature supports that quality of patient care and maintenance of patient safety depends in large part on the competence of the nursing staff with respect to assessment skills and recognition of patients who are acutely ill (Lees \& Hughes, 2009). Furthermore, withdrawal of antiepileptic medications and other provocative factors; place patients admitted to an EMU at particularly high risk for injury (NAEC, 2010, Buelow, Privitera, Levishohn, \& Barkley, 2009). It is critical that nurses who work on an EMU are prepared to accurately assess patients having clinical events and provide a safe environment (AANN, 2007).

\section{Problem Statement}

Diagnosing seizures accurately and in a timely manner depends largely on a thorough assessment of what occurred before, during, and after the event since not all behavior changes are electrographic seizures (Shafer, 1999). Nurses who witness clinical events can add crucial information to the diagnostic phase (Shafer, 1999). As stated in the introduction, there is little evidence in the literature that addresses nursing care of patients on an epilepsy monitoring unit. Provocative factors such as the withdrawal of medication, sleep deprivation, and photic stimulation can produce seizures which may become frequent and severe. Seizure activity may lead to status epilepticus which is a medical emergency (Behrouz, Chen \& Tatum, 2009). Nurses' assessment skills in the EMU are a critical aspect of performing safe and quality care. 
Therefore, it is important that nurses have a good foundation in the application of these skills and that assessments are performed accurately and on a consistent basis. It is essential that nurses are provided with a structured educational program that both assesses their level of competence and provides specific education to improve those skills.

\section{Theoretical Framework}

Patricia Benner's work provided the theoretical framework for this project. Benner's nursing theory, From Novice to Expert, was first published in 1982. The theory was derived from data obtained from the interviews of nurses who described the clinical situations in which they took part. Benner describes nurses as progressing in a linear fashion through five levels of nursing expertise. Benner's theory was based on the Dreyfus Model of Skill Acquisition (as cited in Benner, 2001, p. 13). The five levels of nursing expertise are novice, advanced beginner, competent, proficient and expert.

Novice nurses operate by following rules (rule governed behavior) to guide their practice (Benner, 1982). Since the epilepsy monitoring unit (EMU) where this project took place is a new service to the hospital, the nurses who work on the EMU can be considered novices as they have no prior experience in an EMU. Nurses at the advanced beginner stage on the EMU can, after 1.5 years of experience, use established protocols to make consistent assessments of the patients who are experiencing clinical events. For example, the nurse could walk into the room when a patient is having a clinical event, and if they are following the protocols correctly, will ask the patient to follow a motor command (raise your hand), or ask the patient orientation questions (tell me where you are, tell me your name). At this stage nurses still rely on protocols, as they have not 
experienced enough clinical situations to translate this experience into action (consistent assessment with each clinical event).

As the nurse enters the competent stage of expertise, he/she is better organized and plans his/her time (Benner, 1982). On the EMU on which the study was conducted, it is the policy that epilepsy patients are to be cared for by nurses who have a nurse-to-patient ratio of no more than one to four. Since the unit is equipped with three private rooms that are $100 \%$ operational, the nurse caring for the epilepsy patient on this unit would still have an additional non-monitored patient assigned during his or her shift. It is imperative that nurses who have reached the competent level of nursing expertise on the unit possess and consistently practice good organizational, planning, and assessment skills.

According to Benner (2001), as nurses gain confidence through better organization and planning, they will move into the proficient stage of nursing care. For example, the nurse on the epilepsy monitoring unit who has reached the proficient stage of development would begin to anticipate how the patient will respond based on changes in medication or other changes to the treatment plan as recommended by the epilepsy care team.

In the last stage of development, the expert stage, the nurse is much more intuitive and arrives at solutions in a more expeditious manner (Benner, 2001). Education along with experience is a critical element in nurses acquiring greater skill in caring for patients (Redfern, Norman, Calman, Watson, \& Murrells, 2002). For example, on the EMU nurses at the expert stage of development would anticipate complications that would arise from withdrawal of antiepileptic drugs and would monitor that patient more closely. 
Using Benner's From Novice to Expert model of development to structure an educational program to accommodate each stage of nursing expertise would ensure that the needs of the nurse and patient could be met in an organized and step-wise fashion to improve patient outcomes. Please refer to Appendix B for a diagrammatic explanation of the application of Benner's framework to this proposal. 


\section{Chapter II Review of Literature}

\section{Search Strategy}

The review of literature for this project was completed by searching databases that included Academic Search Complete, EBSCO host, National Guidelines Clearinghouse, the Cochrane Library, CINAHL, and Medline. Language was limited to articles printed in English. Date of publication and country were not limited. Age of participants was restricted to patients over the age of 18 in accordance with inclusion/exclusion criteria for this project. Using the search terms "education" "nurse", "epilepsy" and "monitoring” only eight hits were obtained, with one appropriate article identified. Substituting the word "protocol" for education resulted in four hits with one appropriate article. Next, the terms "nurse", "assessment", "seizures" were used which resulted in 55 hits with one appropriate article identified and two Clinical Practice Guidelines (CPG). The search term "skills" was substituted for "assessment" which resulted in 754 hits. Abstracts were reviewed and articles were excluded if they did not pertain to acute care. Eight appropriate articles were identified, one of which was a systematic literature review. A total of two CPG's, one systematic review, and seven articles were reviewed.

\section{Critical Appraisal}

\section{Epilepsy}

One of the two CPG's identified originated from the American Association of Neurosciences Nurses (AANN) in 2007 and is the second edition in print. This guideline provides general recommendations regarding assessment and monitoring of the patient with seizures. Recommendations for assessment include keeping the patient safe, and observing and 
recording the event as it progresses. The $\mathrm{CPG}$ also recommends providing information on how the seizure started, the location and duration of motor activity, and "any other pertinent details that might assist in the diagnosis of seizure type" (AANN, 2007, p. 9). Post-ictally, the guideline recommends evaluation of motor strength, memory, language and orientation. Using the Appraisal of Guidelines for Research and Evaluation (AGREE) instrument (The AGREE Collaboration, 2001); there are both strengths and weaknesses attributed to the CPG. Strengths include specifically described objectives, rationale, and assessment of scientific evidence. Other strengths include identification of target audience and clearly described criteria for evidence selection. Weaknesses of the CPG include lack of patient's views and preferences, lack of supporting tools for application, and lack of independence from a funding body.

The second clinical practice guideline was put forth by the National Association of Epilepsy Centers (NAEC) in 2010. The guideline discusses the necessary elements for specialized epilepsy centers across the United States. Elements specific to continuous video electroencephalographic monitoring include the use of protocols, especially for medication withdrawal, examination of the patient during a seizure, and measures to be taken if the number, duration, or severity of seizures is excessive. Using the AGREE instrument for appraisal (The AGREE Collaboration, 2001); the guideline's strengths include the definition of patients to whom the guidelines are meant to apply, definition of the target users, specific and unambiguous recommendations, the use of systematic methods to search for evidence, and the provision of a procedure for updating the guideline. Weaknesses include the absence of clearly defined clinical questions, the lack of inclusion of patients' views and preferences, inclusion of only physicians in the guideline development and the lack of identification of cost implications for implementation of the recommendations. 
Smallwood, Wallin, \& Kamensek (1993) evaluated the use of a nursing protocol on the care of patients with seizures. The study used a descriptive design and identified the effectiveness of the protocol on patient outcomes. The sample was selected from patients who had a history of seizures admitted to a neurological unit in a hospital in Vancouver, British Columbia. The study used a prospective sample group who were admitted to the neurological unit for a minimum of 72 hours and later discharged to home. A group was then selected for the purpose of conducting chart audits retrospectively as a comparison for the prospective group. The sample size in the prospective part of the study was 55 with $49 \%$ males and $51 \%$ females included in the sample, and the retrospective sample consisted of 73 patients with the same gender distribution as the prospective sample. Four assessment measures were used in the study: a quality assurance chart audit, a seizure questionnaire, a patient satisfaction survey, and a staff satisfaction survey. A protocol was designed and implemented and a laminated pocket card was distributed to all nurses on the unit with assessment and documentation guidelines. The protocol was designed to provide direction for nurses at all competency levels. Independent ttests, paired t-tests and chi-square analyses were used to evaluate the efficacy of the protocol. The study found that compliance with documentation standards rose from $37.5 \%$ prior to implementation of the protocol to $76.6 \%$ after implementation $(p<.001)$. Patient knowledge regarding their seizure disorder pre-and post-implementation was compared. Patients were more knowledgeable about factors that influence seizure control, medications, definitions of seizures, and diagnostic tests after teaching by nurses regarding these issues. Patient satisfaction results indicated that $83.3 \%$ felt prepared to go home, $98.1 \%$ received enough information from nurses regarding their seizures, and $88.5 \%$ received enough information from doctors. Seventy-three percent learned how to manage a seizure, $73.5 \%$ learned more about their medications, and 
$61.2 \%$ learned information related to lifestyle modifications. Only 38\% increased their knowledge of stress management. In the staff satisfaction survey, $95 \%$ of the nurses viewed the protocol as being a useful guide for assessment, while $85.7 \%$ felt the protocol was a useful guide for intervention. Strengths of the study included a quasi-experimental design, statistical analysis of data, and a prospective and retrospective sample. Weaknesses include small sample size and vague outcome measures such as staff/patient satisfaction.

Baker et al., (2003) studied the utility of neurological assessments of patients in a cardiothoracic surgery intensive care unit to detect neurologic deficits after cardiac surgery. Nurses used the Neurologic Intensive Care Evaluation (NICE) tool to assess patients using a scoring scale from 0 (absent brainstem reflexes) to 8 (oriented). Assessments were performed every halfhour for the first five hours immediately post-surgery. A total of 35 patients were studied. This study demonstrated that the time to reach the lower NICE scores, which reflected mainly brainstem function, was independent of whether the patient suffered from post-operative neurocognitive dysfunction. However, the time required for a patient to reach a NICE scores greater than four, which reflect mainly cortical cognitive function, were prolonged in patients with postoperative neuro-cognitive dysfunction. This single center study concluded that using standardized serial nursing neurologic assessments of post-operative cardiac patients may be a useful tool for early identification of patients with neurologic injury. Strengths of the study included use of statistical analysis and use of an instrument with high inter-rater reliability. Weaknesses include small sample size and single center study.

Buelow, Privitera, Levishohn, \& Barkley (2009) described current practices in epilepsy monitoring units. Investigators developed surveys consisting of 17 questions that were sent to 
physicians who practice in an EMU. A second survey (26 questions) was sent to nurses who practiced in an EMU. Issues such as protocols for medication withdrawal and rescue procedures, patient observers, and safety concerns were addressed in the surveys. Seventeen percent of the physicians and $36 \%$ of the nurses responded. Forty-eight percent of the respondents indicated that medications were never withdrawn prior to admission to the unit while $41 \%$ reported that with some patients, medications were withdrawn prior to admission. In addition, $59 \%$ of the respondents indicated they had written protocols for treatments of seizures compared to $41 \%$ of the respondents who indicated they had no written protocols for treatment of seizures. The respondents were evenly divided as to whether they had the capability of reviewing EEG's from home. Finally, $86 \%$ of the nurse respondents answered that the nursing staff had policies in place with respect to epilepsy monitoring while $8 \%$ indicated they did not have specific policies in place and $6 \%$ were not sure whether there were policies in place regarding epilepsy monitoring. From these and other responses to the survey, the survey demonstrated that there was not a clear consensus of practices among respondents. The conclusion was that there was a wide variation in practice patterns among the EMUs. The authors noted that safety concerns were raised due to a lack of consensus among practices in EMUs across the country. This study has a number of strengths. First, the authors are well respected experts in the field of epilepsy management. Second, the surveys contained open-ended questions which allowed qualitative data to be gathered. Third, separate questions for physicians and nurses allowed specific concerns from both disciplines to be examined. The article does conclude that most EMU's individualize their care for epilepsy patients, and the article does go on to recommend interdisciplinary collaboration to "create a culture of safety" (p. 313). Weaknesses of the article include low 
response rate, limited statistical analysis and no mechanism to exclude responses of multiple practitioners from the same institution.

Shafer (1999) discussed advances in seizure assessment, treatment, and self-management. The article lists specific resources for nurses and patients in the management of epilepsy and includes a comprehensive seizure assessment algorithm from the first edition of the American Association of Neuroscience Nurses Clinical Practice Guideline published in 1999. The CPG recommended assessing level of consciousness inter-ictally as opposed to post-ictally. The algorithm recommends assessing motor commands, orientation, and cognition. The algorithm suggests airway management and checking for injury in patients who are unresponsive. In addition the algorithm includes information regarding the treatment of acute seizures and status epilepticus. The article is written by a single author not well known in the field of epilepsy. The use of a CPG from a recognized society lends credibility to the article. Other obvious weaknesses are lack of experimental design, lack of evidence from descriptive or qualitative studies and references that do not include RCT's or large cohort studies. A strength of the article is the inclusion of specific nursing implications for epilepsy patient care, especially in the area of education for self-management.

\section{Theoretical Model}

Redfern and colleagues (2002) conducted a systematic review assessing competence to practice nursing in the workplace. The workplace was not specifically defined. Analysis of methods of assessing competence to practice nursing included questionnaire rating scales (3 studies), ratings by observation (6 studies), reflection in and on practice (10 studies), selfassessment (4 studies) and multi-method approaches (3 studies). The studies reviewed included 
criterion-referenced rating scales (5 studies), Benner's model of skill acquisition ( 3 studies) and objective structured clinical examination (OSCE) (9 studies). The review found that questionnaire rating scales used to assess competence in nursing were not rigorously tested for reliability and validity. The review concluded that questionnaire rating scales lack adequate reliability and validity testing but does state that "the methodological limitations of observation can be overcome with the use of criterion-referenced scales or simulations such as the OSCE" (p. 56). Observation in the real-life setting was found to have strong support as an assessment of competence according to the review. The review also concludes that using a multi-method approach adds to validity and helps to establish a comprehensive assessment of skills required to practice in nursing. The systematic review is thorough and examines a comprehensive network of learning methods. The review was conducted in universities and workplaces in the United Kingdom, however, the methods of learning that were analyzed were used in other countries which included the USA, Australia, and Scotland making the findings generalizable.

Lees \& Hughes (2009) examined the development of assessment skills of nurses working on an acute medical unit (AMU) in a hospital in Birmingham, England. The purpose of the development and implementation of the framework was to improve clinical expertise among nurses working on the AMU with acutely ill patients. The framework used letters of the alphabet to encompass elements of assessment. The authors noted "the maintenance of patient safety depended on the competence and confidence of the nursing team with regard to assessing and recognizing acutely ill patients" (p. 36). The framework (ABCDEfG) consisted of the following assessment areas: Airway/oxygen therapy, breathing rate, pattern and oxygenation saturation, circulation (blood pressure, pulse and capillary refill time), disability (neurological impairment (awake, verbally responsive, pain responsive, unconscious), exposure (check limbs for edema 
and rashes) and fG (Don't forget glucose). The nurses were educated on using the framework and six months after the introduction of the framework, a retrospective review of nursing notes was conducted. Thirty-four medical records were surveyed. Twenty-five (74\%) of the records showed successful use of the framework and nine (26\%) did not. Four categories were used to quantify the quality of the assessment content: Excellent, good, average and poor. Fifteen records were rated as excellent, eight as good, two as average, and none as poor. The sample consisted of twelve nurses who had between 5 and 15 years experience. The study was a single center study. Strengths of the study included a good variety of teaching strategies and use of a systematic framework. Weaknesses include very small sample size, lack of experimental design and limited statistical analysis.

A descriptive study by Taylor (2002) was published as part of a larger study which evaluated differences between expert and novice nurses in assessing healthcare needs of patients. The study reviewed how novice and expert nurses access information before implementing a nursing procedure. Participants were undergraduate baccalaureate students (novices) and nurses in acute medical-surgical and rehabilitation hospitals with greater than 5 years experience (experts). Observation of participants performing selected procedures and in-depth semistructured interviews were the methods of data collection. Eighty sets of data were collected and 33 sets were used in the final analysis $(n=33)$. Observation of participants performing procedures such showering patients, taking blood pressure, testing a urine sample, and taking a blood glucose measurement were used. An in-depth semi-structured interview was also used in the data collection. 
When preparing to carry out a procedure, nurses sought information from four main data sources which included nursing handover, patient documentation, previous knowledge of the patient and selection of other sources grouped as "miscellaneous". Results showed that all 33 participants had accessed at least one source of preparatory data; however, expert nurses were more apt to access multiple sources of data as compared to novice nurses (Taylor, 2002). A strength of the study was that the process of data collection was precisely described. Limitations included a small sample size and lack of statistical analysis.

In a descriptive observational study (Uitterhoeve et al., 2007), five oncology nurses interviewed an actor playing the role of a patient diagnosed with cancer. Seven different interviews were undertaken by the nurses and these interviews were used to describe nursepatient interactions. The aim of this study was to describe the nurses' response to cues given by the patient (actor) and to gain an understanding of the way nurses address patients' social and emotional needs. Videotaping allowed for rating the interactions using the Medical Interview Aural Rating Scale (MIARS). The findings of this study revealed that half of the patients' cues were responded to with distancing behaviors by the nurses. The other half of the cues were responded to by exploration (33\%) or acknowledging (17\%) behavior. The authors concluded that responding to patients' cues of emotional concerns or worries by acknowledging and/or exploring their feelings was a way to optimize nurse-patient communication. Strengths of this study include the use of videotapes to improve accuracy and the use of software to ensure consistent coding of conversations. Weaknesses include single center study and the use of an actor rather than a real patient which may influence nurses' responses. 


\section{Synthesis of Literature Review}

There is very little information in the literature that specifically addresses nursing care on epilepsy monitoring units. In addition, there is poor consensus about general practice on epilepsy monitoring units (Buelow, Privitera, Levishon \& Barkley, 2009). There is however, strong evidence from two CPGs (NAEC, 2010; AANN, 2007), one systematic review (Redfern, Normal, Calman, Watson \& Murrells, 2002), five descriptive design studies (Uitterhoeve, et al, 2007; Baker, et al. 2003; Taylor, 2002; Lees \& Hughes, 2009; Smallwood, Wallin, \& Kamensek, 1993) and one expert review (Shafer, 1999) to indicate that complete and accurate assessment of patients and their needs is important to providing patient safety and favorable outcomes. Also, there is evidence to support the use of protocols to ensure patient safety and the use of videotapes for accurate assessment of nurse-patient interactions.

It is clear that based on the literature, further research is needed in this area, most notably in the form of prospective studies. The use of protocols is also a common theme identified in the literature related to the care of seizure patients. Rigorous studies with better scientific design that specifically explore nursing care on an EMU will help to guide and inform better evidence based management of patients admitted to epilepsy monitoring units. Therefore the purpose of this capstone was to evaluate the effectiveness of a structured educational program on nurses' assessment rating in an epilepsy monitoring unit.

\section{Congruence of Organization's Strategic Plan to Project}

As in most healthcare organizations across the country, patient safety and satisfaction are important goals (Vahey et. al., 2004). These goals are also important to the institution where the 
capstone project was implemented. The capstone project was in direct alignment with these goals. As stated in the synthesis of the literature review, safety is a general and common concern regarding patients who are acutely ill and of specific concern to patients admitted to epilepsy monitoring units. National guidelines including those put forth by the AANN (2007) and the NAEC ( 2010) discuss the important role of nurses who provide care to patients with seizures. The goal of this project was to improve nurses' assessments of patients on the EMU with the hope that this would lead to improved quality of care for those patients as evidenced by better quantitative scores on the Nursing Assessment Rating Scale tool. 


\section{Chapter III Methodology}

\section{Project Design}

This study used a before and after design to evaluate the effectiveness of a structured educational program on nurses' assessments. Videotapes were reviewed prior to the program and then again after the implementation of the program. An investigator developed tool, the Nursing Assessment Rating Scale (described below), was used to quantitatively measure the interactions and scores comparing pre and post education. There were two objectives for this project. The first objective was to measure nurses' assessments of patients on the EMU in a quantifiable form. The second objective was to evaluate the effectiveness of an educational program on the scores on the Nursing Assessment Rating Scale.

\section{Educational Program}

The educational component of this project was evidence based and addressed antiepileptic drugs, interactions with other commonly used medications, and signs and symptoms of drug toxicity or drug interactions, status epilepticus, and safety (NAEC, 2010, Buelow, Privitera, Levisohn \& Barkley, 2009, AANN, 2007). The educational component was designed to improve nursing assessments of patients during a clinical event.

The program consisted of three different methods and took place over four sessions which lasted approximately 45 minutes. All but two of the twenty-five nurses working on the EMU attended the educational sessions. During those sessions, a power point presentation emphasized first a review of the protocols for care of patients on the EMU that had been in place for two years. This was followed by a discussion of the specific points of patient evaluation that 
was required. This included the neurologic exam, vital signs etc. The necessity of providing a safe environment was also discussed.

After the didactic portion, three case based scenarios were presented with an opportunity for interaction between the epilepsy nurse practitioner and staff nurses. The elements of the nursing assessment were referenced throughout the educational session as evidenced in the table below:

Table 1

Number of Referenced Elements in Educational Program

\begin{tabular}{lc}
\hline Element of Assessment & \# of Times Referenced \\
\hline Response Time & 2 \\
Neuro Exam & 6 \\
Provide Safe Environment & 7 \\
Vital Signs & 3 \\
Pushes Event Button & 2 \\
Respect/Compassion & 2 \\
Appropriate Conversation & 3 \\
\hline
\end{tabular}

Application of Patricia Benner's (1982) framework from Novice to Expert informed the education through the use of multimodal teaching strategies to take advantage of the linear progression of movement through the stages of expertise as identified by Benner. For example, novice nurses practice through the use of rule governed behavior (Benner, 1982) therefore, it was identified that reinforcement of existing protocols would guide novice nurses in assessing patients experiencing clinical events. 
The use of didactic lectures for advanced beginner and competent stages, nurses built upon and refined the materials previously mastered (lists, protocols). Nurses at the advanced beginner and competent stages used previously learned material to apply old and new knowledge to begin to prioritize and plan their patient care. Using the example of instruction on antiepileptic drugs and signs and symptoms of toxicity, the advanced beginner and competent nurse were taught to differentiate between mild dilantin toxicity which may result in nystagmus, and more significant dilantin toxicity which can result in ataxia and falls.

The use of case-based scenarios engaged the nurses in simulated decision-making exercises. Problem or case based learning is an effective teaching tool that can improve critical thinking skills which can then be used in an actual patient situation when it is encountered (Heinrichs, 2002). Case based or problem based learning is an effective teaching strategy for the proficient and expert nurse as they can apply both education and clinical experience to their practice. Case based scenarios that were used in this education helped nurses differentiate between epileptic and non-epileptic events and identify risk factors for specific epilepsy types.

An investigator developed tool, The Nursing Assessment Rating Scale (Appendix A) based upon the Slater Nursing Competencies Rating Scale (Wandelt \& Stewart, 1975) and the Performance Evaluation Tool-Registered Nurse Medical/Surgical Unit (Kostopoulos, 1988), was used to rate assessments. Assessments of the patients were measured using established criteria for the elements of the assessments along with responses using a Likert scale that ranged from unsatisfactory to superior. Criterion based rating scales were more reliable and accurate than those scales who did not use specified criteria as seen in a literature review (Redfern, Norman, Calman, Watson \& Murrells, 2002). The Nursing Assessment Rating Scale was adapted and 
modified from the Slater Nursing Competencies Rating Scale (Wandelt \& Stewart, 1975) and the Performance Evaluation Tool-Registered Nurse Medical/Surgical Unit (Kostopoulos, 1988).

Video clips of patients who have clinical events while admitted to the EMU were reviewed and scored according to the criterion listed beside each element of the assessment. Videos were viewed and scored by an experienced epilepsy nurse practitioner and a graduate student who received instruction on the scoring criteria. Inter-rater reliability of this tool was established with a Cronbach $\alpha$ of .72 and a kappa score of .48. The responses were then entered into a database and tallied according to the number of each response (ex. 25 superior responses for "obtains and assess vital signs"). The same information was again collected and analyzed after the educational program was implemented.

\section{Project Timeline}

The timeline originally established in the Capstone Proposal estimated a dissemination of findings in March of 2011. There were delays getting started with data collection as the project had not been formally approved by the WVU Institutional Review Board. Data collection began immediately after approval. The remainder of the project timeline is found in Appendix D.

\section{Resources/Budget}

In addition to the EMU that is equipped with private rooms, video camera and computer software and hardware to provide monitoring of patients, and nursing assessments and interventions, there were several resources required for this project. First, a reliable and valid measuring tool was necessary to quantify nurses' assessments of patients both pre and post 
intervention. The Nursing Assessment Rating Scale (Appendix A) will be discussed in the results section of this paper.

Second, experts in the content area of the project and personnel familiar with research design and statistical analysis were used to assist with data collection and analysis. The content expert was established and the expert's credentials and experience in the field of epilepsy were well defined.

Third, educational materials were designed and constructed for use in the project. The protocols that were already in place were revisited and reinforced. The protocols were added to another didactic lecture as well as a case-based learning exercise.

Finally, data entry personnel, software upgrades, and miscellaneous office supplies were required for the project. Funding was divided between Cabell Huntington Hospital and Marshall University Department of Neuroscience. Data entry personnel were the most costly of the resources. The data entry took approximately 50 hours. A graduate student from Marshall University assisted with data entry and was paid $\$ 360.00$ for their time.

\section{Evidence of Key Site Support}

Cabell Huntington Hospital/Marshall University Epilepsy Center is the site where the capstone project was implemented. Verbal and written support from the nurse manager on the unit who reports directly to hospital and nursing administration is evidenced in Appendix $\mathrm{C}$ of this paper. Both hospital and nursing administration are committed to quality patient care and patient safety and were in full support of the additional education and staff time the project required. Additionally, the medical director of the epilepsy center and support personnel 
(advanced practice nurse, EEG technologists, and clerical support) also provided support of this project.

\section{Stakeholders}

The stakeholders in this project included staff nurses, hospital, and nursing administration. Additional stakeholders of this project consisted of the epilepsy care team, capstone chair and committee, faculty, and most importantly, patients and families.

\section{Planned Evaluation}

\section{Hypothesis}

Nurses' assessment skills will be improved as evidenced by improved scores on The Nursing Assessment Rating Scale after receiving appropriate educational instruction after receiving an educational program.

\section{Data Collection/Analysis}

The epilepsy monitoring unit nursing quality assessment process provides information on multiple aspects of the nursing care provided with each event as outlined on the data collection sheets in Appendix A. In this data collection sheet there were seven primary quality indicators that assumed the values: unsatisfactory, satisfactory, good and superior. The criteria for each score appear in the appropriate box.

The primary independent variable in the analysis was the nursing assessment performed before and after the educational session. The educational program was designed based on the nurses' level of experience using the conceptual framework of Patricia Benner's From Novice to 
Expert (1982). Elements of the educational program included protocols, traditional didactic lectures and case based discussions. It is noted by Manley and McCormack (2003) that "innovative ways of supporting practice development are required if clinical expertise among nurses is to be developed (p. 26). Assessments were measured again after the nurses attended the educational program. Data were then collected and analyzed again to determine if the education resulted in more consistent assessments as evidenced by improved scores on the Nurse Assessment Rating Scale.

\section{Inclusion/Exclusion Criteria}

Inclusion criteria consisted of data from all adult patients 18 years of age and older admitted to the Cabell-Huntington Hospital/Marshall University epilepsy monitoring unit who had a clinical event while on video-EEG monitoring. Only data from significant clinical events were reviewed.

Exclusion criteria included: patients aged less than 18 years were excluded as care of these patients would have required different sets of nursing skills than care of adult patients. Patients with no data on nursing interactions entered into the quality assessment clinical data base were also excluded. Clinical events in which the epilepsy nurse practitioner was present were also excluded as only staff nurse assessments were analyzed. 


\section{Chapter IV Results}

There are a number of elements that form the background for the data collected for this project. These elements include both patient and nurse demographics.

\section{EMU Patient Demographics}

A total of 36 patients were admitted to the EMU since December 16, 2010. Age, gender, ethnicity and insurance breakdown are represented in table two below:

Table 2

EMU Patient Demographics

\begin{tabular}{cccc}
\hline Age & Gender & Ethnicity* & Insurance \\
& & & \\
\hline $\begin{array}{c}\text { Maximum } \\
\text { Age }=69\end{array}$ & $40 \%$ Female & $\begin{array}{c}85 \% \\
\text { Caucasian }\end{array}$ & $\begin{array}{c}35 \% \\
\text { Medicare }\end{array}$ \\
& & $\begin{array}{c}10 \% \text { African } \\
\text { American }\end{array}$ & $\begin{array}{c}45 \% \\
\text { Medicaid }\end{array}$ \\
Mean & $60 \%$ Male & $5 \%$ Other & $20 \%$ Other \\
Age $=45$ & & & \\
S.D. $=14$ & & & \\
\hline N $=36 . *$ Percentages are estimates of ethnic breakdown
\end{tabular}

$\mathrm{N}=36$. *Percentages are estimates of ethnic breakdown

\section{Nurse Characteristics}

There were 25 nurses working on the EMU at the time of this study. Their demographic information is summarized in Table 3 below: 
Table 3

EMU Nursing Demographics

\begin{tabular}{ccclcc}
\hline Age & Gender & Credential & Degree & $\begin{array}{c}\text { Years of } \\
\text { Experience }\end{array}$ & $\begin{array}{c}\text { \# CNRN } \\
\text { Credential }\end{array}$ \\
\hline $\mathrm{X}=49$ & $81 \%$ Female & $\begin{array}{c}\mathrm{RN}=21 \\
(84 \%)\end{array}$ & $\mathrm{BS}=3(14 \%)$ & $\begin{array}{c}\text { Range of } \\
\text { Experience }\end{array}$ & 4 \\
Range= 24-62 & $19 \%$ Male & $\begin{array}{c}\mathrm{APN}=4 \\
(16 \%)\end{array}$ & & $0-15 \mathrm{yrs}$ & \\
& & & & \\
\hline
\end{tabular}

$\mathrm{N}=25$

\section{Pre-Education Nurse Assessment Rating Scale Scores}

A total of 130 nurse-patient encounters were analyzed as part this study. The quality of all analyzed videos was sufficient to ensure a clear picture of the events. Only encounters from nurses who received the education were included. One hundred encounters were done before the education and 20 were evaluated after the education. Prior to the educational program being delivered to the nurses working on the unit, the mean score on the Nursing Assessment Rating scale was $14.5(\mathrm{SD}=2.1$.) The breakdown of individual elements of the nursing assessment and mean scores along with standard deviation are listed in Table 4.

The multiple t-tests shown in Table 4 demonstrate that the statistically significant changes in the rating scales were in the areas of the neurological examination and respect as suggested by the data shown in Figure 1. Table 4 also indicates that at baseline the highest scores are in the areas of response time and vital signs. The areas of assessment that showed lower 
scores were appropriate conversation and providing a safe environment. These scores showed no changes in time before the education.

Table 4

Pre and Post Education Rating Scale Scores.

\begin{tabular}{|c|c|c|c|c|c|c|c|}
\hline & \multicolumn{2}{|c|}{$\begin{array}{c}\text { Pre } \\
\text { Education }\end{array}$} & \multicolumn{2}{|c|}{$\begin{array}{c}\text { Post } \\
\text { Education }\end{array}$} & \multicolumn{3}{|c|}{ t-tests } \\
\hline Assessment Element & Mean & S.D. & Mean & S.D. & $\mathrm{t}$ & $\mathrm{df}$ & $\mathrm{p}$ \\
\hline Response Time & 2.5 & 0.52 & 2.20 & 0.41 & 2.44 & 128 & 0.015 \\
\hline Neuro Exam & 2.1 & 0.88 & 2.70 & 0.47 & -2.77 & 128 & $0.006^{*}$ \\
\hline Safe Environment & 1.6 & 0.81 & 1.95 & 0.69 & -1.62 & 128 & 0.11 \\
\hline Vital Signs & 2.5 & 0.70 & 2.90 & 0.31 & -2.56 & 128 & 0.011 \\
\hline Event Button & 2.3 & 0.47 & 2.60 & 0.50 & -2.26 & 128 & 0.025 \\
\hline Respect & 2.1 & 0.63 & 2.55 & 0.51 & -3.21 & 128 & $0.0016^{*}$ \\
\hline Conversation & 1.3 & 0.83 & 1.40 & 0.68 & -0.32 & 128 & 0.75 \\
\hline Sum & 14.5 & 2.1 & 16.30 & 1.17 & -3.73 & 128 & $0.0003 *$ \\
\hline
\end{tabular}

Note.*Asterisks indicate $\mathrm{p}$ values that are statistically significant after correction for multiple testing. 
Figure 1. Graph Representation of Pre/Post Education Rating Scale Scores

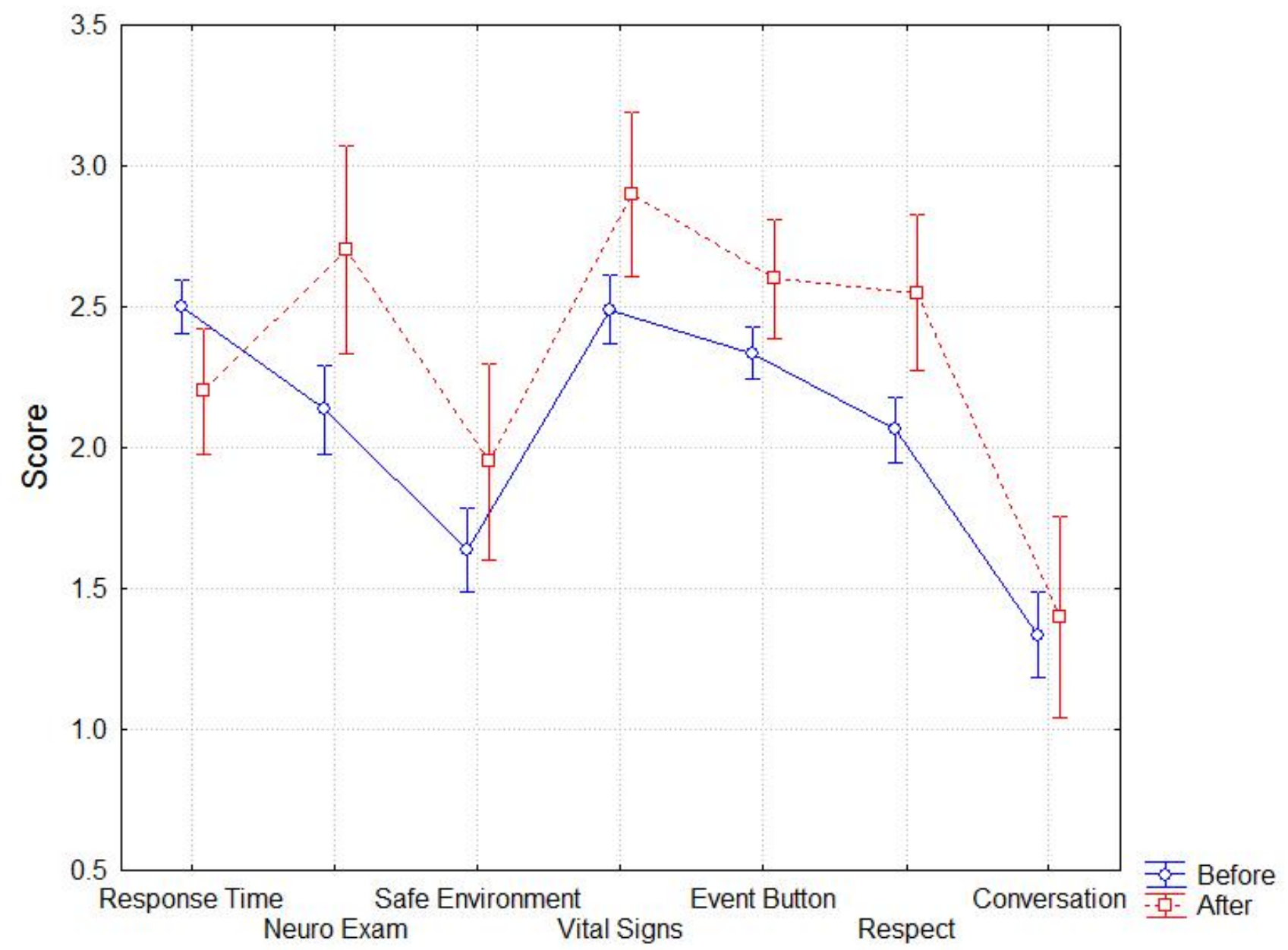

Figure 1. Blue bars represent mean nurse assessment rating scores prior to education. Red bars represent mean scores after education. Statistically significant changes are seen in the neuro exam and respect.

In order to examine what specific aspects of the nursing performance changed in relation to the education, a $2 \times 8$ repeated measures ANOVA was performed with the within subjects variable being the question type ( response time, vital signs, etc.) and the between subjects variable being whether the rating was performed before or after the education. In order to 
confirm statistical significance of the results, multiple t-tests were performed comparing the score for each question before and after the education. Because of multiple tests, a Bonferroni correction was employed and statistical significance was taken for $\mathrm{p}=(.05 / 7)=0.007$ in these $\mathrm{t}$ tests.

Table 5

Pre/Post Education ANOVA Results

\begin{tabular}{llll}
\hline & \multicolumn{2}{c}{ ANOVA Results } \\
\hline Effect & Degrees of Freedom & $\mathrm{F}$ & $\mathrm{P}$ \\
Before/After & $(1,128)$ & $\mathrm{F}(1,128)=13.8$ & $<.01$ \\
Question Type & $(6,768)$ & $\mathrm{F}(6,768)=31$ & $<.01$ \\
Interaction & $(6,768)$ & $\mathrm{F}(6,768)=3.24$ & $<.01$ \\
(Before/After)X(Question & & & \\
Type) & & & \\
\hline
\end{tabular}

Note. Results indicate scores before and after education are different and scores from the different questions are different. Also note the presence of a significant before/after and question type interaction which demonstrates the pattern of the results for each question was different before and after the education. 


\section{Chapter V Discussion and Conclusions}

\section{Discussion}

All of the results in this project were dependent on the instrument used to quantify the nurses' assessments. While no rating scale is perfect, a number of steps were undertaken to improve and test the Nursing Assessment Rating Scale. This included the use of feedback from an initial trial of the instrument to improve the inter-rater reliability of the scores obtained. This resulted in overall moderate agreement between observers that would make the instrument suitable for use. The ability of the instrument to find small changes in the behavior of nurses increased with increasing inter-rater reliability, but the moderate degree of agreement for this instrument was sufficient for this study because the effect of the education was large. In future projects where the effect of an intervention might be smaller, further optimizing of this instrument to obtain a higher kappa score or more measurements may be more helpful.

The quality indicator of response time showed improvement from a mean of 2.5 and a standard deviation of 0.52 to a mean of 2.2 and standard deviation of 0.41 after the educational program. This improvement is significant because prompt assessment allows for timely recognition of life-threatening situations and prevention of adverse outcomes such as status epilepticus (Shafer, 1999).

The most significant improvement was in the area of the neurological exam. Prior to the education, the mean nurse assessment rating scale score was 2.13 with a standard deviation of 0.88. In analyzing the videos prior to the education, the neurological exam was inconsistent and incomplete. After the education was delivered, the rating scale score improved to 2.7 with a 
standard deviation of 0.47 . Nurses consistently assessed level of consciousness and language by asking the patients to follow motor commands (raise your arm, tell me your name, etc.). Accurate assessments are critical to the epilepsy care team in helping to make the correct diagnosis and the neurological exam during a clinical event is a critical component of that assessment. (Shafer, 1999, NAEC, 2010).

Nurses' improvement in the area of providing a safe environment was not statistically significant. Prior to the educational program, the mean score for providing a safe environment was 1.63 with a standard deviation of 0.81 . After the education, the mean rating scale score was 1.95 with a standard deviation of 0.68 . This was not an unexpected finding. Although the educational program provided references regarding the importance of safety seven times (See Table 1), the emphasis on providing a safe environment did not result in a statistically significant improvement. The issue of safety in caring for patients on an EMU is well referenced throughout the literature. The CPG's put forth by the AANN (2007) and the NAEC (2010) make numerous references to the importance of safety in patient care on an EMU. Buelow, Privitera, Levisohn and Barkley, (2009) found that "information to design and establish appropriate practices to improve patient safety in the EMU is scattered, if not entirely lacking" (p.313). As a result of this project, modifications were made to the original protocols established with the inception of the EMU to specifically address and improve the safety of the environment on the EMU.

There was further improvement in the Vital Signs quality indicator from 2.49 to 2.90 with standard deviations of 0.70 and 0.31 respectively. Prior to the education, nurses on the EMU were consistently measuring vital signs of the patients and subsequent to the education improved this element of assessment even further. Because measuring vital signs is an integral component 
of any nursing assessment, nurses are already adept at this skill therefore, the improvement was not statistically significant.

Nurses also improved slightly in ensuring the event button was pushed when patients had a clinical event. The event button marks the electroencephalogram (EEG) so that this area is reviewed first by the epilepsy care team. The mean rating scale score prior to education was 2.33 with a standard deviation of 0.47 . After the education the mean score was 2.6 with a standard deviation of 0.50 . This improvement also was not statistically significant.

There was a statistically significant improvement in the area of respect. Mean rating scale scores in this indicator were 2.06 with a standard deviation of 0.63 . After the education, rating scale scores rose to a mean of 2.55 and a standard deviation of 0.51 . The improvement evidenced in this element of assessment is important because patients are responsive to nurses who provide adequate responses to their concerns (Uitterhoeve et al., 2007).

Finally, there was not a statistically significant improvement in the element of appropriate conversation. Mean scores before the education were 1.33 with a standard deviation of 0.83 . After the education, mean scores were 1.40 with a standard deviation of 0.68 . It is possible this finding may mean that appropriate conversation may be more effectively taught through the use of different methods rather than through case based exercises or didactic lectures.

The findings that resulted from the educational program showed that while the original framework used for this project was a solid foundation, it is apparent that the learning process is much more complex. Benner (1982), addresses levels of competence as a linear progression. 
This project has shown that the many factors that are associated with nursing skill are independent of one another, and competency in any one area may not indicate competency in another area. The data shows that while nurses are consistent in measuring vital signs, they are not consistent in providing a safe environment.

While the multimodal process of education was helpful, modifications to the educational program may be necessary. For example, areas of the assessment that did not significantly improve after the education was delivered such as safety, pushes event button and appropriate conversation, may need to be referenced with more frequency the next time the education is provided and/or taught through simulation exercises.

It is important to note that the absolute value of the scorers obtained in this project do not necessarily reflect the competence, or lack thereof, of nursing care. In fact, providing healthcare in today's society, regardless of specialty, is enormously complicated. The amount of learning, as well as the continuation of that learning on an ongoing basis, is necessary regardless of discipline. The project was designed to examine the most effective teaching strategies for nurses working on the EMU.

\section{Conclusions}

There were three primary conclusions reached a result of this project. First, the Nurse Assessment Rating Scale transformed a qualitative measure of nursing assessment into a quantitative indicator that could be compared pre-and post-education. By assigning a numeric value to rating of superior, good, satisfactory, or unsatisfactory, a quantitative score can be compared to ascertain whether the education did improve scores. 
Second, the data showed that education is important and makes a difference in the way nurses care for patients. Certainly, the nurses were aware of the protocols required for assessments of patients having clinical events. However, there were variations on the exact elements of assessments being performed. Once the original protocols were reinforced and other modalities of education were introduced assessments were more consistent and scores improved.

Third, different modes of education, that is protocols, didactic lectures, and case based scenarios were effective because the specific effect of the education in each area of performance (such as respect and compassion) may not be best learned in a didactic lecture, but rather may be learned more effectively in case based scenarios. The theoretical framework of Benner (1982) is partially supported in this conclusion. While the education did not specifically separate groups of nurses into groups of novices, advanced beginners or experts, the elements of the education were delivered in such a way as to optimize the best ways to learn for these groups; that is protocols, lectures and case based scenarios as suggested by Redfern and colleagues (2002).

For the future, this project shows that there is an opportunity for more study on the effects of an educational program for nurses working on epilepsy monitoring unit. For example, it would be beneficial to examine how long the effects of the education persisted. What would be the implications if those scores declined over time? Should education be given more frequently, or perhaps, should the modalities of the education change? Should the groups be split into novice, advanced beginner, proficient, and expert and the education delivered strictly based on the nurses' level of experience instead of interwoven through all levels of experience? Finally, the practice change of using education that optimizes different levels of nursing experience can 
be carried forward and used not only for educating nurses in the EMU, but also for any care setting including ambulatory or acute care.

\section{Limitations}

While no serious limitations were identified, a few limitations did exist. For example, findings were based on a single center and data were collected retrospectively. Additionally, inter-rater reliability of the data collection instrument could have been higher since the kappa score of 0.48 indicated only moderate agreement. 


\section{References}

American Association of Neurosciences, (2007). Care of the patient with seizures $\left(2^{\text {nd }} e d.\right)$. Retrieved from: http://www.guideline.gov/summary/summary.aspx? $\mathrm{id}=13579 \& \mathrm{nbr}=6951$.

Baker, S., Beauchamp, K, Ballinghoff, J., Escherich, A., Cheung, A.T., \& Stecker, M.M., (2003). Utility of the neurologic intensive cre evaluation (NICE) in detecting neurologic deficit after cardiac operations-a pilot study. Medical Science Monitor, 9, 203-208.

Benner, P, (1982). From novice to expert. American Journal of Nursing, 3, 402-7.

Benner, P. (2001). From novice to expert. (Commemorative ed.). Upper Saddle River NJ: Prentice-Hall, Inc.

Behrouz, R., Chen, S., \& Tatum, W.O., (4 $\left.{ }^{\text {th }}\right)$ (2009). Evaluation and management of status epilepticus in the neurological intensive care unit, Journal of the American Osteopathic Association, 109, 237-245.

Buelow, J.M., Privitera, M., Levisohn, P., \& Barkley, G.L., (2009). A description of current practice in epilepsy monitoring units. Epilepsy \& Behavior, 15, 308-313.

Epilepsy Foundation (2010) Retrieved from http://www.epilepsyfoundtion.org/about /statistics.cfm. 
Heinrichs, K. I., (2002). Problem based learning in entry level athletic training professional educational training programs: A model for developing critical-thinking and decisionmaking skills. Journal of Athletic Training, 37, S189-S198.

Kostopoulos, M.R., (1988). Performance evaluation tool-Registered Nurse, medical/surgical unit [Abstract]. Retrieved from EBSCOhost.

Lees, L., \& Hughes, T. (2009). Implementing a patient assessment framework in acute care. Nursing Standard, 24. 35-43.

Manley, K. \& McCormack, B. (2003). Practice development: purpose, methodology, facilitation, and evaluation. Nursing in Critical Care, 8, 22-29.

National Association of Epilepsy Centers (2010). Guidelines for essential services, personnel, and facilities. ( $3^{\text {rd }}$ ed.). Retrieved from http://www.naec-epilepsy.org/spec_care/ documents/NAEC-FinalGuidelineswithruralcenterrevision.pdf

Pallin, D. J., Goldstein, J. N., Moussally, J. S., Pelleteir, A. J., Green, A. R., \& Camargo, C. A., Jr. (2008). Seizure visits in U.S. emergency departments: epidemiology and potential disparities in care. International Journal of Emergency Medicine, 1, 97-105.

Redfern, S., Norman, I., Calman, L., Watson, R., \& Murrells, T. (2002). Assessing competence to practice in nursing: A review of the literature. Research Papers in Education, 17, 5177.

Shafer, P. (1999). Epilepsy and seizures: Advancement in seizure assessment, treatment, and self-management. Nursing Clinics of North America, 34, 743-759. 
Smallwood, P., Wallin, J., \& Kamensek, J. (1993). Evaluation of a protocol on the care and management of a neurological patient with seizures. Axon, March, 67-71.

Taylor, C. (2002). Assessing patients' needs: Does the same information guide expert and novice nurses? International Nursing Review, 49, 11-19.

The AGREE Collaboration. The Appraisal of Guidelines for Research \& Evaluation (AGREE) Instrument, 2001. London: The AGREE Research Trust.

Uitterhoeve, R., deLeeuw, J., Bensing, J., Heaven, C., Borm, G., deMulder, P., \& van Achterberg, T. (2007). Cue responding behaviors of oncology nurses in video-simulated interviews. Journal of Advanced Nursing, 61, 71-80.

Vahey, D. C., Aiken, L. H., Sloane, D. M., Clarke, S. P., \& Vargas, D. (2004). Nurse burnout and patient satisfaction. Medical Care, 42, 57-66.

Wandelt, M.A., \& Stewart, D.S., (1975). Slater nursing competencies rating scale [Abstract]. Retrieved July 17, 2010 from EBSCOhost.

West Virginia Department of Health and Human Resources (2002). Supplement to the West Virginia county health profiles. Retrieved from http://www.wvdhhr.org/bph/oehp/ hsc/prof2002/cpf11_00.htm.

World Health Organization (2009). Fact Sheet 999. Retrieved from http://www.who.int/ mediacentre/factsheets/fs999/en/ 


\section{Appendix A}

Nursing Assessment Rating Scale

\begin{tabular}{|c|c|c|c|c|}
\hline & $\begin{array}{l}\text { Superior } \\
3 \text { Points }\end{array}$ & $\begin{array}{l}\text { Good } \\
2 \text { Points }\end{array}$ & $\begin{array}{l}\text { Satisfactory } \\
1 \text { Point }\end{array}$ & $\begin{array}{l}\text { Unsatisfactory } \\
\text { 0 Points }\end{array}$ \\
\hline $\begin{array}{l}\text { 1. Response Time } \\
\begin{array}{l}\text { (sec) } \\
\text { (From Button Press or if } \\
\text { no Button Press from } \\
\text { Clinical Event Onset) }\end{array}\end{array}$ & $<15 \mathrm{sec}$ & $15-60 \mathrm{sec}$ & $60-120 \mathrm{sec}$ & $>120 \mathrm{sec}$ \\
\hline $\begin{array}{l}\text { 2. Appropriate } \\
\text { Neurological } \\
\text { Examination }\end{array}$ & $\begin{array}{l}\text { 1-Asks pt to follow motor commands } \\
\text { immediately on arrival. } \\
\text { 2-Formally assesses level of } \\
\text { consciousness } \\
\text { 3-Formally assesses language function }\end{array}$ & $\begin{array}{l}\text { Exam delayed more than } 20 \\
\text { seconds or missed one out of } \\
3 \text { factors described under } \\
\text { superior. }\end{array}$ & $\begin{array}{l}\text { Missed at least } 2 \text { of the } \\
\text { factors listed under } \\
\text { superior. }\end{array}$ & No examination performed \\
\hline $\begin{array}{l}\text { 3. Provides Safe } \\
\text { Environment }\end{array}$ & $\begin{array}{l}\text { 1-Turns patient on side as appropriate } \\
\text { 2-Formally assess safety and takes } \\
\text { measures to prevent a fall } \\
\text { 3-Prevents the patient from injuring } \\
\text { himself or herself }\end{array}$ & $\begin{array}{l}\text { Two out of three factors } \\
\text { listed under superior. }\end{array}$ & $\begin{array}{l}\text { One out of three factors } \\
\text { listed under superior. }\end{array}$ & $\begin{array}{l}\text { Attends to none of the } 3 \text { factors } \\
\text { listed under superior performance }\end{array}$ \\
\hline $\begin{array}{l}\text { 4. Obtains and Assesses } \\
\text { Vital Signs }\end{array}$ & $\begin{array}{l}\text { 1-BP } \\
\text { 2-Pulse Oximetry } \\
\text { 3-Fingerstick Glucose } \\
\end{array}$ & $\begin{array}{c}2 \text { of the criteria listed under } \\
\text { Superior }\end{array}$ & $\begin{array}{l}1 \text { of the criteria listed } \\
\text { under Superior. }\end{array}$ & $\begin{array}{c}\text { None of the criteria listed under } \\
\text { Superior. }\end{array}$ \\
\hline $\begin{array}{l}\text { 5. Event button pushed } \\
\text { by nurse if not pushed } \\
\text { by patient }\end{array}$ & $\begin{array}{l}\text { 1-Pushes event button } \\
\text { 2-Gives verbal description of event. } \\
\text { 3-Knows where the alarm cancellation } \\
\text { button is located. }\end{array}$ & $\begin{array}{c}2 \text { of the criteria listed under } \\
\text { Superior }\end{array}$ & $\begin{array}{l}1 \text { of the criteria listed } \\
\text { under Superior. }\end{array}$ & $\begin{array}{c}\text { None of the criteria listed under } \\
\text { Superior. }\end{array}$ \\
\hline $\begin{array}{l}\text { 6. Exhibits Respect and } \\
\text { Compassion }\end{array}$ & $\begin{array}{l}\text { 1-Does not subject patient to painful } \\
\text { stimulation. } \\
\text { 2-Uses respectful language. } \\
\text { 3-Consoles patient after event as } \\
\text { appropriate. }\end{array}$ & $\begin{array}{c}2 \text { of the criteria listed under } \\
\text { Superior }\end{array}$ & $\begin{array}{l}1 \text { of the criteria listed } \\
\text { under Superior. }\end{array}$ & $\begin{array}{c}\text { None of the criteria listed under } \\
\text { Superior. }\end{array}$ \\
\hline $\begin{array}{l}\text { 7. Appropriate } \\
\text { Conversation with } \\
\text { Patient and Family }\end{array}$ & $\begin{array}{l}\text { 1-Addresses Patient by Correct Name } \\
\text { 2-Communicates Appropriate Medical } \\
\text { Information } \\
\text { 3-Explains all actions being taken }\end{array}$ & $\begin{array}{c}2 \text { of the criteria listed under } \\
\text { Superior }\end{array}$ & $\begin{array}{l}1 \text { of the criteria listed } \\
\text { under Superior. }\end{array}$ & $\begin{array}{c}\text { None of the criteria listed under } \\
\text { Superior. }\end{array}$ \\
\hline
\end{tabular}




\section{Appendix B}

\section{Patricia Benner}

From Novice to Expert

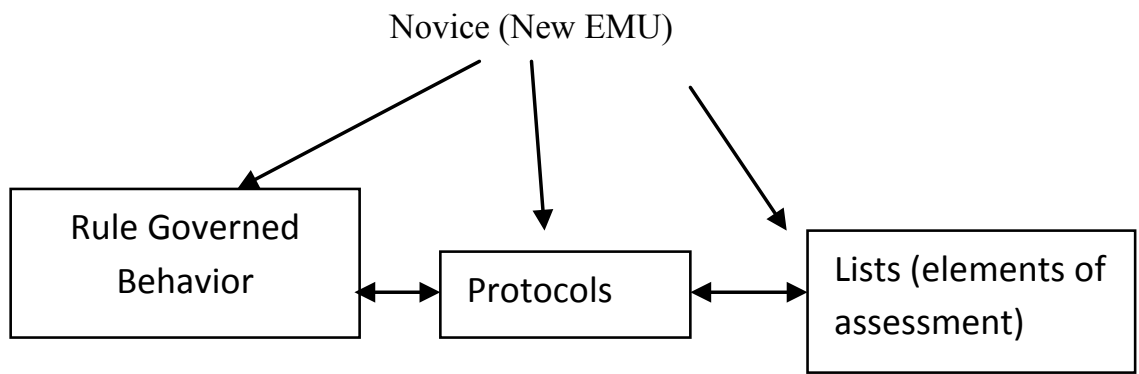

Advanced Beginner (Minimal Experience on EMU)
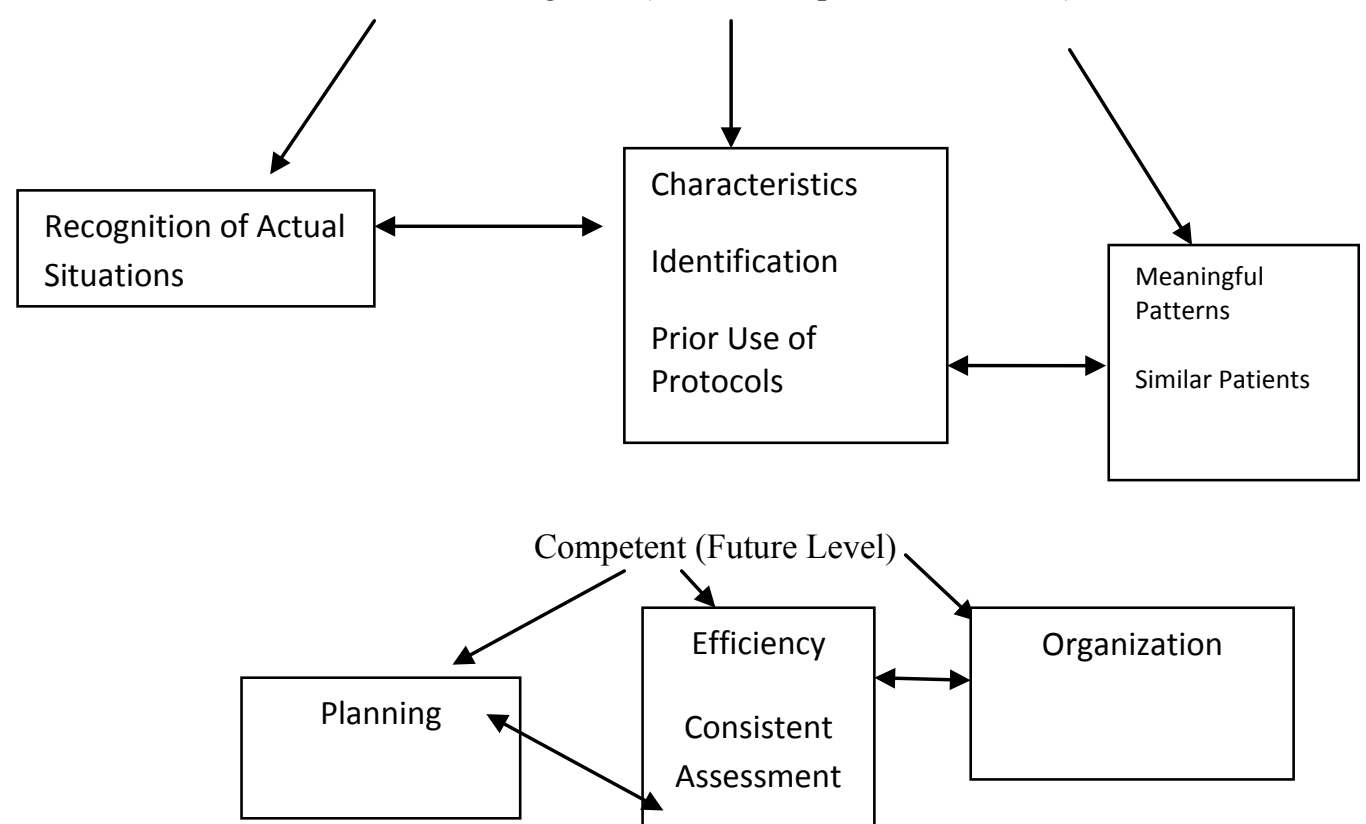

Mona Stecker, 2010

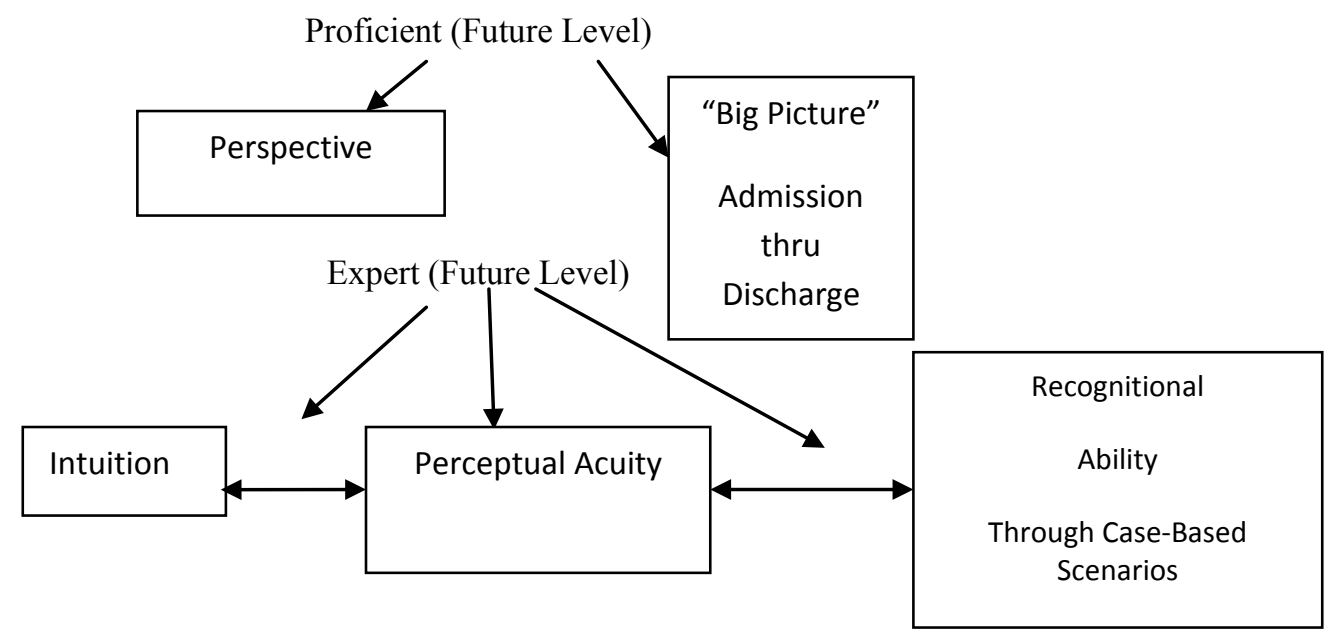




\section{Appendix C}

August 23,2010

To Whom lt May Concern:

Please allow this letter to serve as support of a structured educational program to be implemented on the Epilepsy Monitoring Unit at Cabell Huntington Hospital for the benefit of the nurses and support personnel who are employed on the unit.

Please do not hesitate to contact me if further information is required.

Sincerely,

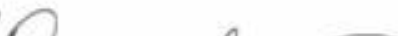

Teresa Sexton, RN, MSNeD

Manager, 4 North

Cabell Huntington Hospital

$1340 \mathrm{Hal}$ Greer Blvd.

Huntington, WV 25701

Phone: $304-399-6731$ 


\section{Appendix D}

Project Timeline

The implementation of the project followed the timeline below

\begin{tabular}{|l|l|}
\hline \multicolumn{1}{|c|}{ Month/Year } & \multicolumn{1}{|c|}{ Activity } \\
\hline October, 2010 & $\begin{array}{l}\text { 1. Capstone proposal approved by } \\
\text { Committee }\end{array}$ \\
\hline November, 2010 & 2. Proposal submitted to WVUIRB \\
\hline December, 2010 & $\begin{array}{l}\text { 1. Developed written and visual } \\
\text { educational materials }\end{array}$ \\
\hline December, 2010 & $\begin{array}{l}\text { 1. Submitted educational materials to } \\
\text { nurse manager EMU/epilepsy center } \\
\text { director for approval }\end{array}$ \\
\hline January, 2011 & $\begin{array}{l}\text { 1. WVUIRB approval of project } \\
\text { 2. Baseline data collected }\end{array}$ \\
\hline February, 2011 & $\begin{array}{l}\text { 1. Educational material revised and } \\
\text { approved by EMU nurse manager and } \\
\text { EMU medical director. }\end{array}$ \\
\hline 2. Baseline data entered and analyzed
\end{tabular}

